\title{
Meiotic pairing and imprinted X chromatin assembly in Caenorhabditis elegans
}

\author{
Christopher J Bean ${ }^{1,3}$, Christine E Schaner ${ }^{1-3}$ \& William G Kelly ${ }^{1}$
}

\begin{abstract}
The genetic imprinting of individual loci or whole chromosomes, as in imprinted X-chromosome inactivation in mammals ${ }^{1,2}$, is established and reset during gametogenesis; defects in this process in the parent can result in disease in the offspring $^{3}$. We describe a sperm-specific chromatin-based imprinting of the $X$ chromosome in the nematode Caenorhabditis elegans that is restricted to histone $\mathrm{H} 3$ modifications. The epigenetic imprint is established during spermatogenesis and its stability in the offspring is affected by the presence of a pairing partner during meiosis in the parental germ line. We observed that DNA lacking a pairing partner during meiosis, the normal situation for the $X$ chromosome in males, is targeted for methylation of histone H3 at Lys9 (H3Lys9) and can be silenced. Targeting unpaired DNA for silencing during meiosis, a potential hallmark of genome defense, could therefore have a conserved role in imprinted X-chromosome inactivation and, ultimately, in sex chromosome evolution.
\end{abstract}

The mechanisms by which imprinting is established during gametogenesis are not understood, but all such epigenetic phenomena clearly involve DNA methylation or histone modifications ${ }^{4-7}$. Methylation of H3-Lys9 is a highly conserved modification that is enriched in facultative and constitutive heterochromatin ${ }^{8}$. Methylation of histone $\mathrm{H} 3$ Lys4 (H3-Lys4), an opposing mark to methylation of H3-Lys9, is even more highly conserved and is enriched in transcriptionally competent or active DNA in all eukaryotes that have been examined ${ }^{8}$. Cytosine methylation, which is not found in all organisms, requires histone methylation in some species ${ }^{9}$, suggesting that histone modification may be the underlying mechanism guiding epigenetic processes. Indeed, methylation of histone $\mathrm{H} 3$ may provide an epigenetic mark during gametogenic imprinting of individual loci ${ }^{10,11}$. Histone modifications established during gametogenesis might therefore create a heritable epigenetic code that controls imprinted transcriptional competency in the offspring.

The X chromosome in early adult germ cells of $C$. elegans lacks histone modifications associated with transcriptional competence ${ }^{12}$. The $\mathrm{X}$ chromosome is silenced in immature gametes of both sexes but shows sex-specific regulation during later gametic stages. In XX hermaphrodites, histones on the $\mathrm{X}$ chromosome become highly modified during early oogenesis, coincident with a burst of X-linked oocyte-specific gene expression ${ }^{12}$. In contrast, the unpaired $\mathrm{X}$ chromosome in (XO) males never accumulates 'activating' modifications during spermatogenesis but becomes enriched in methylated H3Lys 9 and condenses prematurely in pachytene nuclei ${ }^{13}$. This is markedly similar to what is observed for the XY body in mammalian spermatogenesis ${ }^{13}$. The $\mathrm{X}$ chromosome is also depleted of genes encoding sperm-enriched transcripts in C. elegans ${ }^{12,14}$. XX crossprogeny (produced from fertilization of a hermaphrodite by a male) therefore inherit sex chromosomes from each parent that differ substantially both in the spectrum of epigenetic marks established during gametogenesis and in their history of transcriptional activity.

We tested for heritable consequences of these epigenetic differences in cross-progeny (male sired) XX embryos using antibodies specific for individual histone modifications. In one-cell embryos, antibodies against dimethylated H3-Lys4 and diacetylated H3-Lys9/Lys14 (acetylated H3) strongly labeled all chromosomes except one, which was depleted of these epitopes (Fig. 1a,b). The chromosome was unique to the sperm pronucleus, identified by its location opposite to the pole bodies in fertilized oocytes. The sperm pronucleus initially lacked all assayed histone modifications on entering the oocyte (data not shown) but rapidly accumulated them during decondensation, as is observed in mammals ${ }^{15}$. Even at very early stages of decondensation, a large region of the sperm chromatin lacked dimethylated H3Lys4 and acetylated H3 (Fig. 1c), indicating that the chromosome is uniquely prevented from acquiring these modifications, rather than having them specifically removed. This exclusion was heritable through several rounds of DNA replication, with each daughter cell containing a single undermodified chromosome at subsequent cell divisions (Fig. 1d,e). With further cell division, methylated H3-Lys4 and acetylated $\mathrm{H} 3$ accumulated on the undermodified chromosome ( $>12$ cells; Fig. 2 ), and we never observed a unique chromosome in embryos past the 20-cell stage (data not shown).

The epigenetic mark was restricted to absence of the histone $\mathrm{H} 3$ modifications described above. Acetylation of histone H4 (at Lys8 and Lys16) was similar among all chromosomes at all stages examined (data not shown). We detected phosphorylation of H3-Ser10 on all

${ }^{1}$ Biology Department, 1510 Clifton Road, Rollins Research Center and ${ }^{2}$ Graduate Program in Biochemistry, Cell, and Developmental Biology, Emory University, Atlanta, Georgia 30322, USA. ${ }^{3}$ These authors contributed equally to this work. Correspondence should be addressed to W.G.K. (bkelly@biology.emory.edu).

Published online 21 December 2003; doi:10.1038/ng1283 
Figure 1 The $X_{p}$ chromosome lacks a subset of histone $\mathrm{H} 3$ modifications in the early embryo. (a,b) Antibodies to methylated H3Lys4 (a, green) and to acetylated H3 (b, green) are excluded from one chromosome (arrowheads) in one-cell embryos counterstained with DAPI (red). Telomeric regions also appear to exclude these antibodies (small arrows). (c) Exclusion of antibody to dimethylated H3-Lys4 from a large region of the sperm pronucleus shortly after fertilization (arrowhead). (d,e) A single chromosome with reduced staining for antibody to methylated H3-Lys4 persists in two-cell (d) and four-cell (e) embryos (arrowheads). An extruded polar body (marked with an asterisk), visible in $\mathbf{e}$, also stains with this antibody. ( $\mathbf{f}-\mathbf{h})$ Males with an X-integrated GFP transgene were mated to normal XX worms to unambiguously identify the sperm-derived $X$ chromosome $\left(X_{p}\right)$ in crossprogeny $X X$ embryos. In representative nuclei from two-cell (f), four-cell (g) and six-cell

(h) XX cross-progeny embryos, staining with antibody to dimethylated $\mathrm{H} 3$-Lys4 (green) is always absent from the $X_{p}$ chromosome

(arrowheads), marked by DNA FISH specifically targeting the transgenic sequences (blue). The FISH probe labeled approximately $50 \%$ of crossprogeny embryos, identifying XX embryos carrying both maternal and paternal X chromosomes. Red shows DAPI counterstain. Scale bars, $5 \mu$ m.
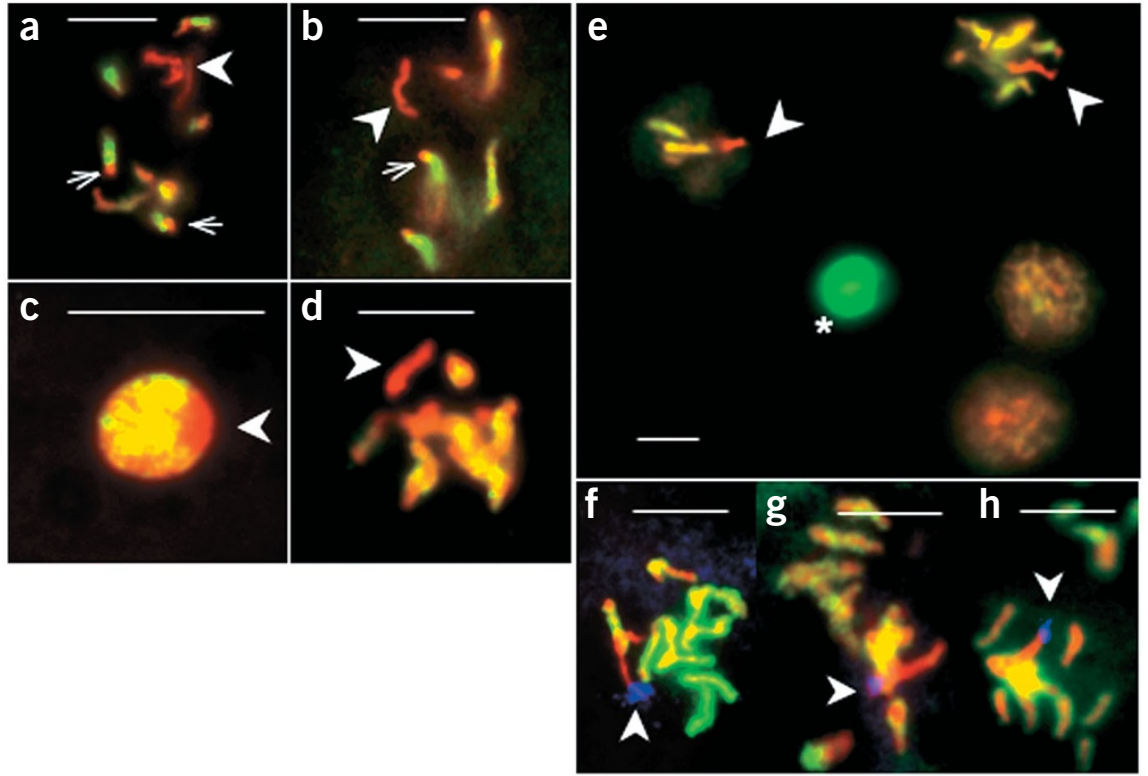

prophase chromosomes, suggesting that bulk cleavage of $\mathrm{H} 3$ tails was unlikely (data not shown). Antibodies specific to trimethylated H3Lys4 showed a pattern of staining similar to that of antibodies to dimethylated H3-Lys4 (data not shown). Methylated H3-Lys9 is present at low levels on all chromosomes in early C. elegans embryos and is mostly enriched at telomeres ${ }^{16}$.

The exceptional chromosome is the sperm-derived $\mathrm{X}$ chromosome $\left(\mathrm{X}_{\mathrm{p}}\right)$, as determined by combined histone antibody staining and DNA fluorescence in situ hybridization (FISH). XO embryos, which normally carry only an oocyte-derived $\mathrm{X}$ chromosome $\left(\mathrm{X}_{\mathrm{m}}\right)$, did not exhibit an undermodified chromosome. An $X_{p}$-specific probe always labeled the undermodified chromosome in XX crossprogeny (Fig. 1f-h). The epigenetic status of the X chromosome is therefore dependent on parental or gamete origin, and thus represents an imprinted 'chromatin state'. We call the undermodified chromosome the 'epigenetically imprinted X chromosome' and the lack of methylated H3-Lys4 and acetylated H3 the 'epigenetic imprint'.

Reproduction in C. elegans occurs predominantly through selffertilization in XX hermaphrodites using stored sperm generated before gametogenesis switches to oogenesis at the adult molt ${ }^{17}$. Therefore, all progeny from self-fertile hermaphrodites, with rare exceptions, are diploid with respect to the $\mathrm{X}$ chromosome. All early-stage selfprogeny from N2 (wild-type) hermaphrodites (N2 (XX)) had a single epigenetically imprinted X chromosome (Fig. 2). We observed this chromosome only in the sperm pronucleus in one-cell embryos (data not shown). The establishment of the X chromatin imprint in C. elegans therefore depends solely on the sex of the gamete: it is established during spermatogenesis in both XO males and XX hermaphrodites.

The stability of the epigenetic imprint in the offspring differed in embryos produced from male sperm versus those from hermaphrodite sperm. Exclusion of methylated H3-Lys4 reversed more quickly in selfprogeny $(\mathrm{N} 2(\mathrm{XX}))$, with an epigenetically imprinted X chromosome frequently indistinguishable by the 10-cell stage and never observed in embryos with more than 14 nuclei (Fig. 2). In contrast, male-sired XX cross-progeny with more than 12-14 nuclei (N2 (XO)) frequently had the epigenetically imprinted $\mathrm{X}$ chromosome (Fig. 2). An $\mathrm{X}_{\mathrm{p}}$ chromosome originating from $\mathrm{XO}$ spermatogenesis therefore has an epigenetic imprint that is more stable than that of an $\mathrm{X}_{\mathrm{p}}$ chromosome from XX spermatogenesis.

The $\mathrm{X}$ chromosome has a pairing partner in the XX hermaphrodite germ line but not in the XO male germ line. In males, the unpaired $\mathrm{X}$ chromosome passes through meiosis in a tightly condensed conformation and becomes enriched for methylated H3-Lys9 during pachynema, similar to the XY sex body that forms during mammalian male meio$\operatorname{sis}^{12,13,18}$. In contrast, during larval hermaphrodite spermatogenesis the paired X chromosomes are not enriched for methylated H3-Lys9 (W.G.K., unpublished observations). We tested whether pairing during

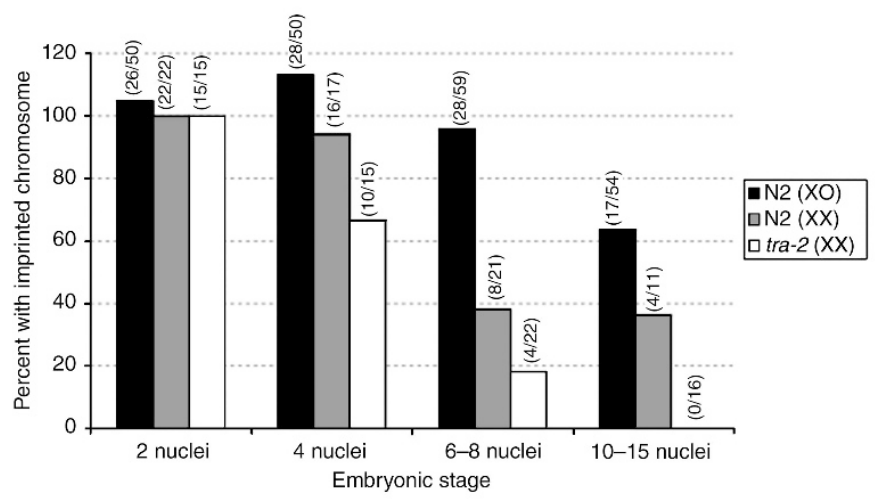

Figure 2 Meiotic pairing in the parent affects the stability of the epigenetic imprint in the offspring. The presence of the epigenetically imprinted $X$ chromosome at different stages (number of nuclei) was compared in offspring from normal XO male crosses (N2 (X0)), tra-2 (q276) XX male crosses (tra-2 (XX)) and N2 hermaphrodite self-progeny (N2 (XX)). The number of embryos optically sectioned and assessed for each class and stage is indicated in parentheses at the top of each bar. 
male spermatogenesis could affect the stability of the epigenetic imprint in XX offspring by carrying out crosses using tra-2 (q276) mutants, which have an XX genotype but develop as fertile males ${ }^{19}$. All offspring sired by these males inherited an $\mathrm{X}_{\mathrm{p}}$ chromosome that went through male meiosis in a paired state. Cross-progeny from tra-2 XX males had even lower stability of the epigenetic imprint than did XX hermaphrodite self-progeny (tra-2 (XX); Fig. 2). The presence or absence of a meiotic pairing partner during spermatogenesis can therefore substantially affect the heritable stability of the $\mathrm{X}_{\mathrm{p}}$ epigenetic imprint in the offspring. The intermediate stability of the epigenetic imprint observed in XX hermaphrodite cross-progeny may reflect unknown influences from the intrinsic differences between male and hermaphrodite spermatogenesis.

Meiotic pairing also affects X-specific chromatin assembly in spermatocytes of tra-2 (q276; XX) males. Methylated H3-Lys4 was absent from the paired X chromosomes in tra-2 (q276) male germ cells at all stages, similar to observations from XO spermatocytes (data not shown). In contrast to XO males, however, the paired X chromosomes in tra-2 (q276) male germ cells were not enriched for methylated H3-Lys9 (Fig. $3 \mathbf{b}, \mathbf{c}$ inset). The pattern of methylation of H3-Lys9 was instead markedly similar to that observed in normal XX oocytes (Fig. 3a) and during normal spermatogenesis in XX L4 larvae (W.G.K., unpublished observations). X-chromosome pairing is therefore dominant over germline sex for targeting of methylated H3-Lys9 in germ cells. This is further illustrated in another sex-determination mutant, her-1 ( $h v 1 y 101)$, in which XO worms develop as hermaphrodites. In her-1 ( hv1y101) XO oocytes, the unpaired X chromosome is transiently enriched for methylated H3-Lys9 and prematurely condenses, similar to what is seen in normal male spermatocytes (Fig. $\mathbf{3 b}, \mathbf{d}$ ). The targeting of methylated H3-Lys9 to the X chromosome in oocytes caused defects in its transcriptional regulation. In normal oogenesis, a late meiotic accumulation of methylated H3-Lys4 on the X chromosome normally occurs coincident with a burst of X-linked gene expression ${ }^{12}$, but this is considerably delayed in her-1 (hv1y101) XO oocytes (Fig. $\mathbf{3 e}-\mathbf{j}$ ), and X-linked oocyte-enriched gene expression is specifically defective (Fig. 3k-n). We also observed no viable self- or cross- progeny from these worms, suggesting that X-chromosome activation during oogenesis is required for fertility (C.E.S. and W.G.K., unpublished results). These results show that, independent of the sex of the germ line, an unpaired X chromosome passing through meiosis acquires a repressive chromatin architecture that is difficult to dismantle.
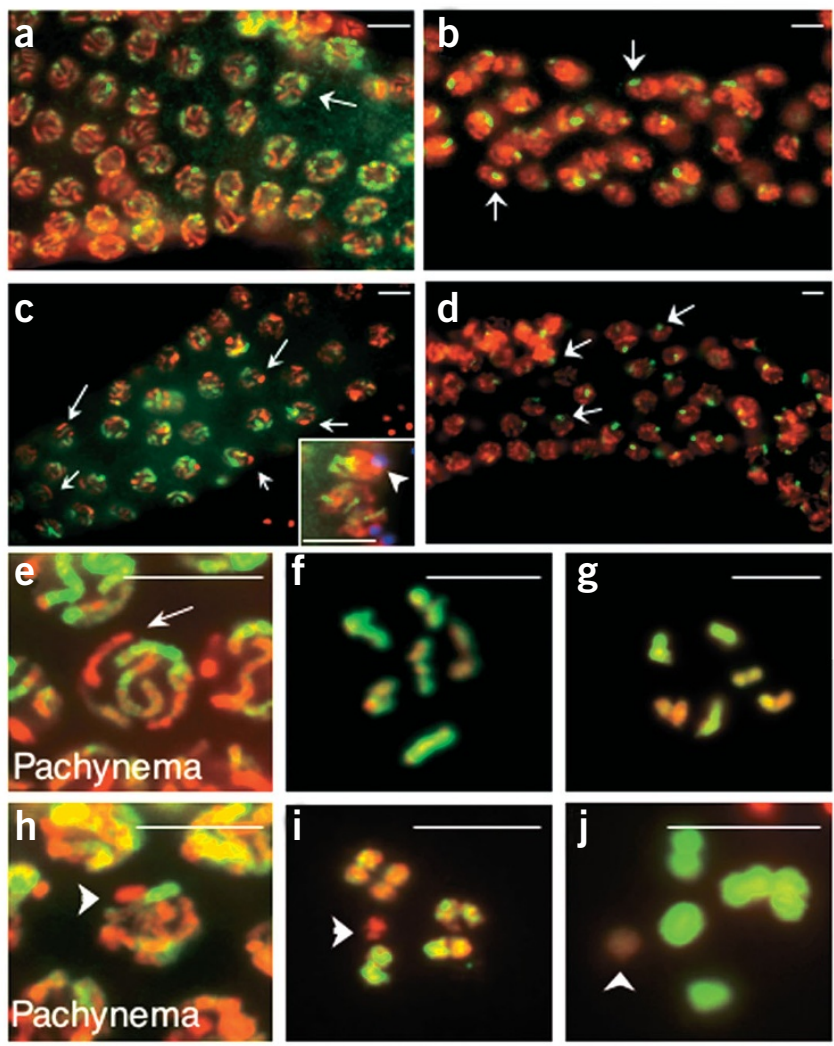

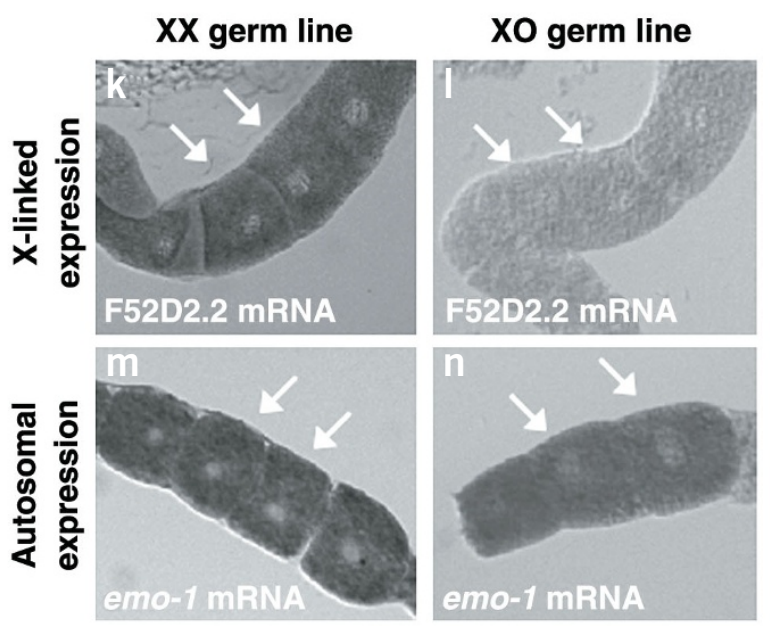

Figure 3 Meiotic targeting for methylation of H3-Lys9 and transcriptional repression of an unpaired X chromosome. (a-d) Staining with antibody to methylated H3-Lys9 (green) in adult gonads counterstained with DAPI (red in all panels). (a) Wild-type N2 (XX) late pachytene-diplotene oocytes. Arrow indicates an individual nucleus with normal enrichment for methylated H3-Lys9 at diplotene stage in oogenesis ${ }^{12}$. (b) N2 (XO) mid-pachytene spermatocytes; arrows indicate X chromosome stained with antibody to methylated H3-Lys9 present in all nuclei. (c) tra-2 (q276) XX late

pachytene-diplotene spermatocytes have a pattern of staining with antibody to methylated H3-Lys9 similar to that of XX oocytes (a). Arrows indicate unstained chromosome in representative nuclei, identified by FISH costaining as the paired X homologs in the inset (arrow; blue shows DNA FISH). (d) her-1 ( hv1y101) XO early- to mid-pachytene oocytes, with arrows indicating unpaired X chromosomes in several nuclei. (e-j) Staining with antibody to methylated H3-Lys4 (green) in N2 (XX) germ cells initially does not label the X chromosome in pachytene cells (arrow in e) but labels all six chromosome pairs in early (f) and late ( $\mathbf{g}$ ) diakinetic oocytes. (h-j) In her-1 XO oocytes, accumulation of methylated H3-Lys4 on the unpaired X chromosome (arrows) is markedly delayed in early (i) and understained in late (j) oocytes. (k-n) Strong mRNA in situ hybridization of a probe for an X-linked oocyte-enriched gene (F52D2.2; ref. 14) is detected in developing oocytes (arrows) of XX her-1 (hv1y101) worms (k), but little or no signal is observed in developing oocytes of XO worms (I). Other X-linked oocyte-enriched genes showed similar defects in transcriptional activity (data not shown). In contrast, mRNA of an autosome-linked oocytespecific gene (emo-1) is easily detected in developing oocytes (arrows) of both XX (m) and XO (n) worms. Scale bars, $5 \mu \mathrm{m}$. 

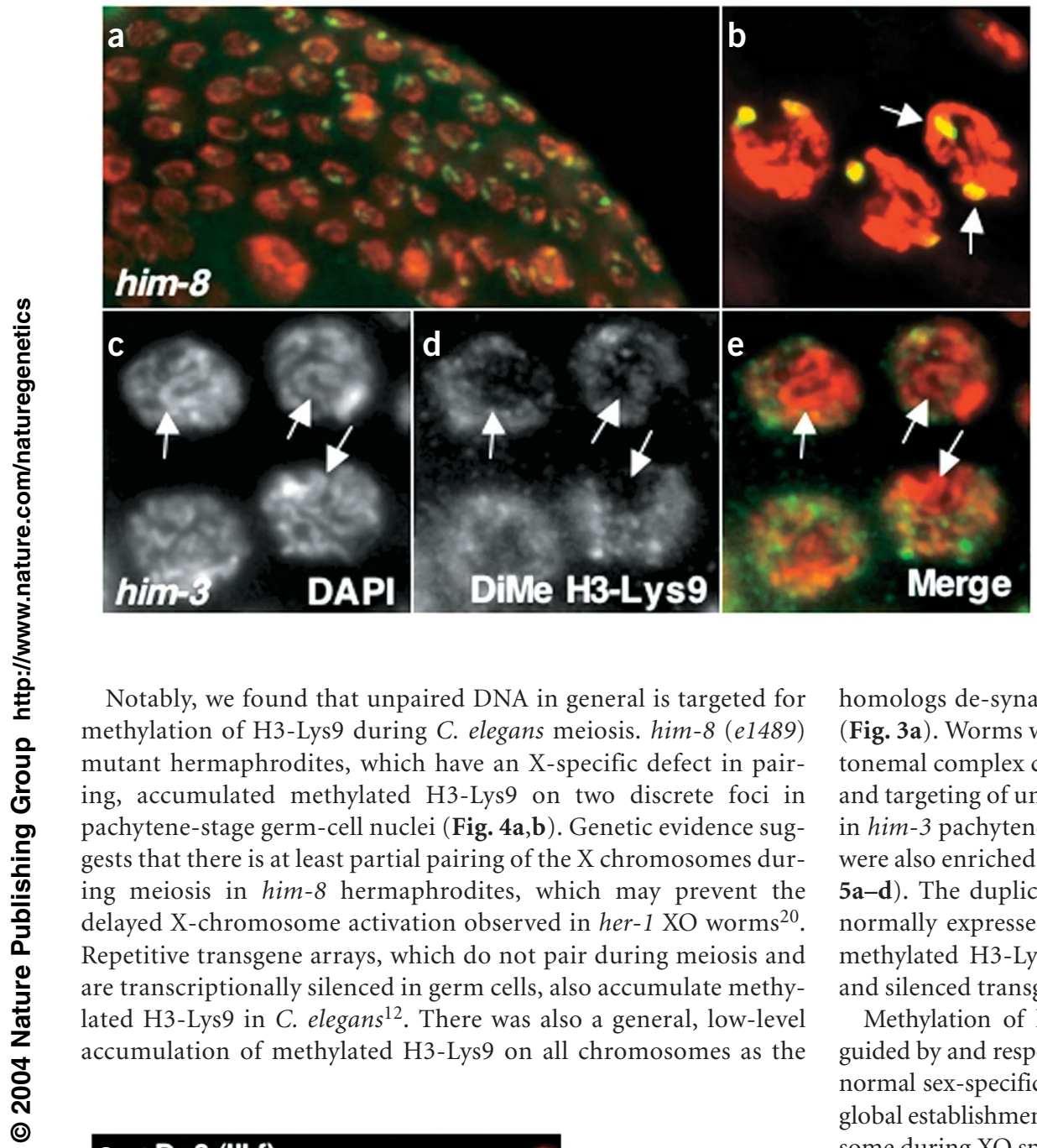

Figure 4 Targeting of methylated H3-Lys9 to the $\mathrm{X}$ chromosome and autosomes is determined by pairing status and not ploidy. (a,b) him-8XX hermaphrodite pachytene-stage germ cells stained for methylated H3-Lys9 (green) have two strong foci of staining, which are presumably the $\mathrm{X}$ chromosomes that are specifically defective in pairing in this mutant. Both chromosomes also lack methylated H3-Lys4 staining (data not shown), which is normal for the $\mathrm{X}$ chromosome in pachynema, but seem to activate normally in diplonema. This is consistent with evidence for partial recombination, and therefore partial pairing, of the $\mathrm{X}$ chromosomes in this mutant. (c-e) Hermaphrodite germ cells defective for HIM-3, a synaptonemal complex component, show widespread staining for methylated H3-Lys9 in nonsynapsed homologs in midpachynema (green in e). In contrast, the few homologs that show pairing in these nuclei did not accumulate methylated H3-Lys9 (arrows). Red shows DAPI counterstain. DiMe H3-Lys9, dimethylated H3-Lys9.
Notably, we found that unpaired DNA in general is targeted for methylation of H3-Lys9 during C. elegans meiosis. him-8 (e1489) mutant hermaphrodites, which have an X-specific defect in pairing, accumulated methylated H3-Lys9 on two discrete foci in pachytene-stage germ-cell nuclei (Fig. 4a,b). Genetic evidence suggests that there is at least partial pairing of the $\mathrm{X}$ chromosomes during meiosis in him- 8 hermaphrodites, which may prevent the delayed X-chromosome activation observed in her-1 XO worms ${ }^{20}$. Repetitive transgene arrays, which do not pair during meiosis and are transcriptionally silenced in germ cells, also accumulate methylated H3-Lys9 in C. elegans ${ }^{12}$. There was also a general, low-level accumulation of methylated H3-Lys9 on all chromosomes as the
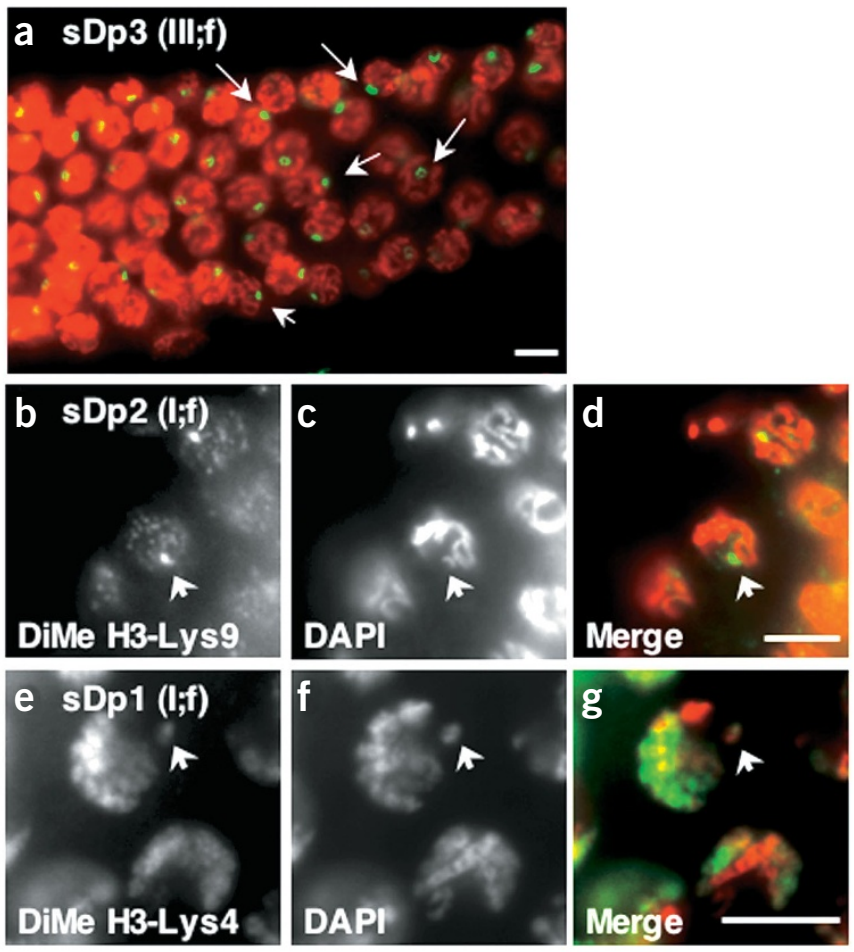

homologs de-synapse at the pachynema-diplonema transition ${ }^{12,16}$ (Fig. 3a). Worms with a mutation in him-3, which encodes a synaptonemal complex component, had defective pairing of all homologs and targeting of unpaired chromosomes for methylation of H3-Lys9 in him-3 pachytene nuclei (Fig. 4c-e). Free autosomal duplications were also enriched for methylated H3-Lys9 in pachytene nuclei (Fig. 5a-d). The duplicated autosomal fragments, which contain genes normally expressed in germ cells, also had detectable amounts of methylated H3-Lys4, in contrast to the unpaired X chromosome and silenced transgene arrays ${ }^{12}$ (Fig. 5e-g).

Methylation of histone $\mathrm{H} 3$ in C. elegans germ cells is therefore guided by and responsive to meiotic pairing, which in turn influences normal sex-specific X chromatin assembly. Our results show that the global establishment of facultative heterochromatin on the X chromosome during $\mathrm{XO}$ spermatogenesis is due primarily to its unpaired status. Enrichment in methylated H3-Lys9, in turn, affects the stability (in the offspring) of a specific gametic epigenetic imprint imposed on the sperm X chromosome. The similarity, in both structure and targeting of methylated H3-Lys9, of the unpaired X chromosome in C. elegans and the poorly paired XY body in mammalian spermatogenesis is notable and may reflect a conserved process ${ }^{13}$. This common process may contribute to the occurrence of an epigenetically imprinted paternal X chromosome observed in female offspring of multiple species. In addition, the general targeting of unpaired DNA for methylation of H3-Lys9 during C. elegans meiosis is reminiscent of meiotic silencing

Figure 5 Unpaired autosomal fragments are also targeted for enrichment for methylated H3-Lys9 in pachytene nuclei. (a) Staining with antibody to methylated H3-Lys9 (green) produces a distinct focus in each pachytene nucleus of a strain carrying the large free autosomal duplication sDp3 (arrows). Red shows DAPI counterstain. (b-d) Antibody to methylated H3Lys9 colocalizes with a cytologically distinguishable example of another unpaired free duplication, sDp2 (arrows). DiMe H3-Lys9, dimethylated H3Lys9. (e-g) sDP1, which labels with antibody to methylated H3-Lys9 (data not shown) can also accumulate dimethylated (DiMe) H3-Lys4 (green) in germ cells (arrowheads). Whether these two modifications colocalize or are mutually exclusive on these duplications is not known; the antibodies used against both marks are both from rabbits and thus difficult to observe simultaneously. A large region of $s D p 1$, readily visible in $\mathbf{g}$, has less staining for methylated H3-Lys4. Scale bars, $5 \mu \mathrm{m}$. 
of unpaired DNA in Neurospora, which is a likely component of genome surveillance ${ }^{21}$. This suggests that genome defense mechanisms may have conserved roles during meiosis in a variety of species. Natural targets for such mechanisms would include new transposon insertions and the highly diverged homologous chromosomes that have evolved in heterogametic species. Conserved genome defense mechanisms could therefore directly influence gene expression in offspring by guiding epigenetic processes in the germ line of the parent.

In the case of the C. elegans X chromosome, the lack of a pairing partner for the male $\mathrm{X}$ chromosome, and its consequential targeting for silencing by potential genome defense mechanisms, could explain the loss of sperm-specific genes from the $\mathrm{X}$ chromosome, as well as those required for both spermatogenesis and oogenesis ${ }^{14}$. Extensive accumulation of methylated H3-Lys9 causes a substantial hindrance to global activation, as illustrated by the impairment of X-linked transcription in her-1 oogenesis (Fig. 3b,d,k,l). Processes involving sexual antagonism could be contributing to sex chromosome evolution, especially in organisms, such as Drosophila, in whom recombination is lacking in males, and in accumulation of female-acting genes on the $\mathrm{X}$ chromosome ${ }^{22,23}$. Pre-existing mechanisms that target heterochromatin assembly onto unpaired DNA in the heterogametic sex, however, could theoretically be sufficient to drive sperm-specific or essential germ-cell genes off the X chromosome as it, and its degenerating or absent pairing partner, evolves. The availability of a pairing partner for the $\mathrm{X}$ chromosome in females would prevent extensive methylation of H3-Lys9 during female meiosis and thus allow oocyte-specific genes to remain on the $\mathrm{X}$ chromosome $\mathrm{e}^{14}$.

The imprinted X chromatin regulation we observe in offspring may be a compounded consequence of both the unpaired status and evolved transcriptional quiescence of the $\mathrm{X}$ chromosome during spermatogenesis. A chromatin status that is heritably refractory to acquiring histone $\mathrm{H} 3$ modifications that correlate with transcriptional competence is assembled, and this assembly is strengthened by methylation of H3-Lys9 when the $\mathrm{X}$ chromosome is unpaired. The evolutionary consequences of these dynamics may explain the paucity of essential early embryonic genes on the $\mathrm{X}$ chromosome in C. elegans ${ }^{24-26}$. Indeed, the small number of such genes may explain the viability of embryos that receive both $\mathrm{X}$ homologs from the female parent $\left(\mathrm{X}_{\mathrm{m}} \mathrm{X}_{\mathrm{m}}\right)$; the consequences of receiving both $\mathrm{X}$ homologs from the male parent $\left(\mathrm{X}_{\mathrm{p}} \mathrm{X}_{\mathrm{p}}\right)$ have not been adequately explored ${ }^{27}$. When uniparental $\mathrm{X}$-chromosome inheritance was investigated in C. elegans, a single viable $\mathrm{X}_{\mathrm{p}} \mathrm{X}_{\mathrm{p}}$ worm was recovered (frequency of $<0.001$ ), which raises the question of whether this worm was a rare 'escaper' ${ }^{27}$. It was recently shown ${ }^{28}$ that under certain conditions XX worms can be transformed into XO males by preferential $\mathrm{X}_{\mathrm{p}}$ loss, underscoring the persistence of epigenetic differences between the two X chromosomes during development that may relate to our results.

The underlying nature of the epigenetic imprint is unclear: possibilities include the specific incorporation of a histone $\mathrm{H} 3$ variant that can not be methylated or acetylated at the residues assayed, or a replacement of histone $\mathrm{H} 3$ by a specific protamine that is targeted to silenced chromatin. In either scenario, non-replication-coupled replacement with canonical histone $\mathrm{H} 3$ isoforms at fertilization may be inefficient or blocked, requiring repeated rounds of replication-coupled histone H3 deposition before modification reaches detectable levels.

In summary, these results support a hypothesis in which genome defense mechanisms, which are normally targeted to unique or unpaired DNA during meiosis, provide an evolutionary drive that not only shapes the genetic profile of the sex chromosome, but may also influence its function during early embryogenesis in a gamete-specific manner. These mechanisms may be conserved and could be driving both sex-chromosome evolution and paternal X-chromosome imprinting in other organisms.

\section{METHODS}

Strains. We used standard techniques for worm maintenance and handling ${ }^{29}$. We carried out all crosses and grew all worms at $20^{\circ} \mathrm{C}$. We used the following strains: wild-type N2 (Bristol), $d p y-5$ (e61) unc-13 (e51) I; sDp1 (I;f), unc-11 (e47) dpy-5 (e61) I; sDp2 (I;f), tra-2 (q122d) II, tra-2 (q276) II, unc-4 (e120) II, dpy-17 (e164) let-718 (s2445) unc-32 (e189) III; sDp3 (III;f), him-8 (e1489) IV, her-1 (hv1y101) V and ccIn4810 (lamin:GFP) X. We generated homozygous tra-2 (q276) XX males by picking male self-progeny from cloned tra-2 (q276) / mnC1 (dpy-10 (e128) unc-52 (e444)) hermaphrodite larvae. To identify the her1 (hv1y101) XO hermaphrodites, we used the following mating scheme. We crossed N2 males to her-1 (hv1y101) unc-42 (e270) V hermaphrodites, and mated resulting non-Unc $\mathrm{F}_{1}$ males to $d p y-11$ (e224), her-1 (hv1y101), unc-42 (e270) V; lon-2 (e678) X hermaphrodites. We collected the resulting Unc nonDpy, Lon progeny as her-1 (hv1y101) XO hermaphrodites.

Immunocytochemistry. We carried out whole-mount fixation and antibody staining of worms and embryos as previously described ${ }^{12}$. For detection of specific histone modifications, we used the following primary antibodies generated in rabbit: antibody to histone $\mathrm{H} 3$ acetylated at Lys9 and Lys14 (Upstate Biotechnology), antibody to dimethylated H3-Lys4, antibody to dimethylated H3-Lys9, antibody to histone H3 phosphorylated at Ser10 (gifts from C. David Allis, University of Virginia), antibody to trimethylated H3-Lys4 (Abcam) and antibodies to histone $\mathrm{H} 4$ acetylated at Lys8 and to histone H4 acetylated at Lys16 (Serotech). We used goat antibody to rabbit IgG conjugated to Alexafluor594 (Molecular Probes) as secondary antibody for visualization.

Scoring. We observed images using a Leica DMRA microscope outfitted with a Cooke Sensicam. We acquired and processed the images with Volume Scan, Microtome (Vaytek) and Image-Pro Plus (Media Cybernetics) software. We determined the presence or absence of an understained chromosome for some experiments by optical sectioning of embryo nuclei at intervals of $0.1-0.2 \mu \mathrm{m}$ and serial analysis of the sections.

We assayed offspring from normal XO male crosses, tra-2 (q276) XX male crosses and N2 hermaphrodite self-progeny for the presence of the epigenetically imprinted X chromosome (Fig. 2). Embryos were staged based on the number of nuclei scored by DAPI staining. To ensure $100 \%$ cross-progeny, we crossed the N2 and tra-2 males with tra-2 $\left(q 122^{\mathrm{d}}\right)$ females, which do not make sperm. Only embryos with a clear absence or presence of the epigenetically imprinted chromosome in (prophase) nuclei were scored. As cell division is asynchronous in these embryos, not all nuclei in a single embryo could be scored simultaneously. We therefore conservatively scored embryos as positive if the epigenetically imprinted chromosome was unambiguously present in the prophase nuclei available to score and as negative if the chromosome was clearly absent or ambiguous (e.g., a partly or faintly stained chromosome). The values for the N2 (XO) matings were corrected for the observed frequency of XO male offspring (0.504), which we determined in parallel crosses (XO male offspring do not contain a sperm-derived X chromosome and thus would not have an epigenetically imprinted X chromosome).

FISH analysis. For combined histone antibody and DNA FISH experiments, we first carried out antibody staining as described above. To process the postsecondary antibody samples for FISH, we next immersed slides in a formaldehyde fixative (3.7\% formaldehyde, $80 \mathrm{mM}$ HEPES buffer ( $\mathrm{pH} 6.9$ ), $1.6 \mathrm{mM}$ MgSO4, $0.8 \mathrm{mM}$ EGTA) for 5-20 min. After a 5-min rinse in distilled water, we fixed samples in 3:1 methanol:glacial acetic acid for $15 \mathrm{~min}$ and then allowed them to air dry.

We carried out FISH using the digoxigenin-labeled (Roche) probe L4054 (Fire Laboratory 1999 Vector Kit) that recognizes the sequence for GFP in transgenic worms. We applied the hybridization solution, consisting of labeled probe in $50 \%$ formamide, $2 \times$ saline sodium citrate (SSC) and $10 \%$ dextran sulfate, to each slide under a glass coverslip and denatured the samples at $80^{\circ} \mathrm{C}$ for $10 \mathrm{~min}$. After an overnight hybridization at $37^{\circ} \mathrm{C}$ in a sealed humid chamber, we washed slides in $50 \%$ formamide, $2 \times \mathrm{SSC}$ at $43^{\circ} \mathrm{C}$ for $15 \mathrm{~min}$, in $2 \times \mathrm{SSC}$ at $43{ }^{\circ} \mathrm{C}$ for $7 \mathrm{~min}$ and in phosphate-buffered saline containing $0.1 \%$ Tween-20 at room temperature for $5 \mathrm{~min}$. We detected hybridized slides with fluoresceinlabeled antibody to digoxigenin (Roche) in $4 \times$ SSC, $3 \%$ bovine serum albumin and $0.1 \%$ Tween-20 for $2 \mathrm{~h}$ at $37^{\circ} \mathrm{C}$ in a humid chamber. After three 10 -min 
washes in phosphate-buffered saline containing $0.1 \%$ Tween-20, we counterstained slides with $5 \mu \mathrm{g} \mathrm{ml}^{-1}$ DAPI (Sigma). We observed the prepared samples and recorded and processed images as described above.

mRNA in situ analyses. We generated DNA probes for two X-linked and two autosomal oocyte-expressed genes as partial cDNAs cloned in pCR2.1 (Invitrogen) from a C. elegans cDNA library. X-linked genes, F52D2.2 and F35D2.2, are located at genetic positions -17.31 and -5.56 , respectively, and are therefore dispersed and unlikely to be subject to local mechanisms of coregulation. $n h r-23$ is located on chromosome I and emo- 1 is located on chromosome $\mathrm{V}$, two dispersed autosomal loci. To generate single-stranded sense and antisense digoxigenin-labeled probes, we carried out PCR with linearized clones and a single primer flanking the insert. Primer sequences are available on request. We fixed and processed dissected gonads from adult hermaphrodites as described elsewhere ${ }^{30}$.

\section{ACKNOWLEDGMENTS}

We thank T. Schedl for helpful suggestions and sharing strains and C. David Allis for sharing antibody reagents. This work was supported by a grant from the US National Institutes of Health (to W.G.K.). C.E.S. is supported by a Training Grant from the US National Institutes of Health. Some of the C. elegans strains used in this study were provided by the Caenorhabditis Genetics Center. Some of the primer sequences used in this study were provided by WormBase.

\section{COMPETING INTERESTS STATEMENT}

The authors declare that they have no competing financial interests.

Received 30 October; accepted 1 December 2003

Published online at http://www.nature.com/naturegenetics/

1. Goto, T. \& Monk, M. Regulation of X-chromosome inactivation in development in mice and humans. Microbiol. Mol. Biol. Rev. 62, 362-378 (1998).

2. Huynh, K.D. \& Lee, J.T. Imprinted X inactivation in eutherians: a model of gametic execution and zygotic relaxation. Curr. Opin. Cell Biol. 13, 690-697 (2001).

3. Reik, W. \& Walter, J. Genomic imprinting: parental influence on the genome. Nat. Rev. Genet. 2, 21-32 (2001).

4. Fournier, C. et al. Allele-specific histone lysine methylation marks regulatory regions at imprinted mouse genes. EMBO J. 21, 6560-6570 (2002).

5. Heard, E. et al. Methylation of histone $\mathrm{H} 3$ at Lys-9 is an early mark on the $\mathrm{X}$ chromosome during $X$ inactivation. Cell 107, 727-738 (2001).

6. Peters, A.H. et al. Histone $\mathrm{H} 3$ lysine 9 methylation is an epigenetic imprint of facultative heterochromatin. Nat. Genet. 30, 77-80 (2002).

7. Boggs, B.A. et al. Differentially methylated forms of histone $\mathrm{H} 3$ show unique association patterns with inactive human X chromosomes. Nat. Genet. 30, 73-76 (2002).

8. Kouzarides, T. Histone methylation in transcriptional control. Curr. Opin. Genet. Dev.
12, 198-209 (2002).

9. Tamaru, H. \& Selker, E.U. A histone H3 methyltransferase controls DNA methylation in Neurospora crassa. Nature 414, 277-283 (2001).

10. Xin, Z., Allis, C.D. \& Wagstaff, J. Parent-specific complementary patterns of histone $\mathrm{H} 3$ lysine 9 and $\mathrm{H} 3$ lysine 4 methylation at the Prader-Willi syndrome imprinting center. Am. J. Hum. Genet. 69, 1389-1394 (2001).

11. Higashimoto, K. et al. Loss of CpG methylation is strongly correlated with loss of histone $\mathrm{H} 3$ lysine 9 methylation at DMR-LIT1 in patients with Beckwith-Wiedemann syndrome. Am. J. Hum. Genet. 73, 948-956 (2003).

12. Kelly, W.G. et al. X-chromosome silencing in the germline of C. elegans. Development 129, 479-492 (2002)

13. Cowell, I.G. et al. Heterochromatin, HP1 and methylation at lysine 9 of histone H3 in animals. Chromosoma 111, 22-36 (2002).

14. Reinke, V. et al. A global profile of germline gene expression in C. elegans. Mol. Cell 6, 605-616 (2000)

15. Adenot, P.G., Mercier, Y., Renard, J.P. \& Thompson, E.M. Differential H4 acetylation of paternal and maternal chromatin precedes DNA replication and differential transcriptional activity in pronuclei of 1 -cell mouse embryos. Development 124 4615-4625 (1997)

16. Reuben, M. \& Lin, R. Germline X chromosomes exhibit contrasting patterns of histone H3 methylation in Caenorhabditis elegans. Dev. Biol. 245, 71-82 (2002)

17. Schedl, T. Germ-line development. in C. elegans // (eds. Riddle, D., Blumenthal, T. Meyer, B. and Priess, J.) 241-269 (Cold Spring Harbor Press, Cold Spring Harbor, New York, 1997).

18. Handel, M.A. \& Hunt, P.A. Sex-chromosome pairing and activity during mammalian meiosis. Bioessays 14, 817-822 (1992)

19. Kuwabara, P.E. \& Perry, M.D. It ain't over till it's ova: germline sex determination in $C$. elegans. Bioessays 23, 596-604 (2001).

20. Broverman, S.A. \& Meneely, P.M. Meiotic mutants that cause a polar decrease in recombination on the $\mathrm{X}$ chromosome in Caenorhabditis elegans. Genetics 136 119-127 (1994)

21. Hynes, M.J. \& Todd, R.B. Detection of unpaired DNA at meiosis results in RNA-mediated silencing. Bioessays 25, 99-103 (2003).

22. Wu, C.I. \& Yujun Xu, E. Sexual antagonism and X inactivation - the SAXI hypothesis. Trends Genet. 19, 243-247 (2003).

23. Parisi, M. et al. Paucity of genes on the Drosophila $X$ chromosome showing malebiased expression. Science 299, 697-700 (2003).

24. Baugh, L.R., Hill, A.A., Slonim, D.K., Brown, E.L. \& Hunter, C.P. Composition and dynamics of the Caenorhabditis elegans early embryonic transcriptome. Development 130, 889-900 (2003)

25. Piano, F. et al. Gene clustering based on RNAi phenotypes of ovary-enriched genes in C. elegans. Curr. Biol. 12, 1959-1964 (2002).

26. Kamath, R.S. et al. Systematic functional analysis of the Caenorhabditis elegans genome using RNAi. Nature 421, 231-237 (2003).

27. Hodgkin, J., Horvitz, H.R. \& Brenner, S. Nondisjunction mutants of the nematode Caenorhabditis elegans. Genetics 91, 67-94 (1979).

28. Prahlad, V., Pilgrim, D. \& Goodwin, E.B. Roles for mating and environment in C. elegans sex determination. Science 302, 1046-1049 (2003)

29. Brenner, S. The genetics of Caenorhabditis elegans. Genetics 77, 71-94 (1974).

30. Seydoux, G. \& Fire, A. Soma-germline asymmetry in the distributions of embryonic RNAs in Caenorhabditis elegans. Development 120, 2823-2834 (1994). 